\title{
GERAÇÃO SONOQUÍMICA DE OXIDANTES EM SOLUÇÃO AQUOSA SATURADA DE TETRACLORETO DE CARBONO
}

\author{
Sivanildo da Silva Borges \\ Instituto de Química, Universidade Federal da Bahia, Campus Universitário de Ondina, 40170-290 Salvador - BA \\ Mauro Korn* \\ Departamento de Ciências Exatas e da Terra, Universidade do Estado da Bahia, Estrada das Barreiras s/n, 41195-001 Salvador - BA
}

Recebido em 11/6/01; aceito em 8/11/01

\begin{abstract}
SONOCHEMICAL PRODUCTION OF OXIDIZING SPECIES IN WATER SOLUTION SATURATED WITH CARBON TETRACHLORIDE. In this work the $\mathrm{CCl}_{4}$ degradation in aqueous solution by sonication with $40 \mathrm{kHz}$ commercial ultrasonic bath was investigated. Sonochemical degradation of $\mathrm{CCl}_{4}$ occur by the cleavage of $\mathrm{C}-\mathrm{Cl}$ bond into the cavitation bubbles. Oxidation reactions and the $\mathrm{pH}$ decreasing in the bulk solution during sonication were attributed to chlorine radicals produced by $\mathrm{CCl}_{4}$ sonolysis, leading to increase the chloride concentration. The formation of oxidizing agents was evaluated employing $\mathrm{I}^{-}$and $\mathrm{Fe}^{2+}$ ion solutions, converted to $\mathrm{I}_{2}$ and $\mathrm{HIO}$, and $\mathrm{Fe}^{3+}$, respectively. The amount of chloride and hydronium ions produced after 3 min of irradiation was 11.52 and $12.19 \mathrm{mmol}$, respectively, suggesting that the same reaction was involved to produce these ions. $\mathrm{Fe}^{2+}$ oxidation and the $\mathrm{pH}$ variation were monitored to estimate chlorine radical formation rate in the presence $\left(0.107 \mu \mathrm{mol} \mathrm{s}{ }^{-1}\right)$ and absence $\left(0.092 \mu \mathrm{mol} \mathrm{s} \mathrm{s}^{-1}\right)$ of metallic ion during the first minute of sonication.
\end{abstract}

Keywords: sonochemical reactions; oxidizing species generation; carbon tetrachloride sonolysis.

\section{INTRODUÇÃO}

Os efeitos químicos provocados pelos ultra-sons ocorrem devido ao fenômeno da cavitação acústica, que é o processo de nucleação, crescimento e colapso de bolhas transientes em líquidos expostos a ondas ultra-sônicas de baixa frequência $(<1 \mathrm{MHz})^{1}$. As ondas ultrasônicas se propagam através de um líquido em ciclos alternados de compressão e expansão, porém, caso a onda acústica tenha pressão suficientemente alta, pode vencer as forças intermoleculares num ponto do líquido, criando uma cavidade para a qual podem se difundir, durante a etapa de expansão, os gases e vapores presentes no líquido. $\mathrm{Na}$ etapa de compressão os gases e vapores não retornam completamente para o líquido, resultando em aumento efetivo do tamanho da cavidade durante os ciclos seguintes de compressão e expansão até que seja atingido seu diâmetro crítico, entrando em violento colapso ${ }^{2}$. O colapso das bolhas transientes provoca a liberação de grande quantidade de energia, gerando temperaturas locais instantâneas muito elevadas $(5200 \mathrm{~K})$ e pressões da ordem de centenas de atmosferas ${ }^{3,4}$.

O processo de cavitação e as temperaturas geradas no colapso são fortemente dependentes da pressão de vapor do solvente. Assim, moléculas de solventes com altas pressões de vapor (e.g. moléculas de água) podem penetrar nas cavidades e, no momento do colapso, sofrerem sonólise, resultando na formação de novos produtos (e.g. $\mathrm{H}_{2}$ e $\mathrm{H}_{2} \mathrm{O}_{2}$ ).

Propriedades físico-químicas dos gases dissolvidos (condutividade térmica, capacidade calorífica, pressão parcial e solubilidade no solvente) garantirão a eficiência da cavitação e, conseqüentemente, da sonólise ${ }^{5,6}$. Em outra aproximação, sabe-se que a eficiência de cavitação depende da freqüência e amplitude das ondas acústicas, potência do sonicador, bem como da tensão superficial, força iônica

\footnotetext{
*e-mail: mkorn@campus1.uneb.br
}

e temperatura do meio solvente ${ }^{7}$. Também, a presença de sólidos favorece à cavitação no meio irradiado ${ }^{8,9}$.

As reações induzidas pelos ultra-sons iniciam-se com a ativação e/ou sonólise das espécies presentes na fase gasosa, através da geração de espécies que podem reagir com outras presentes na mesma fase, na interface gás-líquido ou presentes no líquido. Estas reações devem levar à produção de espécies quimicamente mais estáveis que aquelas formadas pela sonólise. Em se tratando da água, a alta energia gerada pelo colapso de bolha de cavitação, contendo moléculas de água, leva à formação dos radicais $\cdot \mathrm{H}$ e $\bullet \mathrm{OH}$, pela clivagem homolítica de ligações $\mathrm{O}-\mathrm{H}$ das moléculas de água ${ }^{10}$. Estes podem se combinar produzindo água; ou sofrerem arranjos para a formação de $\mathrm{H}_{2}$ e $\mathrm{H}_{2} \mathrm{O}_{2}$. Contudo, outros produtos podem ser formados, caso outras espécies estejam presentes no meio sonicado.

Quando água é sonicada na presença de solvente com maior pressão de vapor, deve ocorrer a sonólise preferencial das moléculas deste. A decomposição sonoquímica de $\mathrm{CCl}_{4}$ em solução aquosa é baseada neste fundamento, ou seja, moléculas de $\mathrm{CCl}_{4}$ migram para as bolhas de cavitação e, devido à alta energia gerada no colapso, as ligações $\mathrm{C}-\mathrm{Cl}$ são homoliticamente rompidas, originando os radicais - $\mathrm{CCl}_{3} \mathrm{e} \cdot \mathrm{Cl}$. Os efeitos da sonólise do $\mathrm{CCl}_{4}$ vêm sendo objeto de diversos estudos ${ }^{11-17}$.

$\mathrm{O} \mathrm{CCl}$ e outros solventes orgânicos clorados são empregados em procedimentos de análise química para a solubilização de alguns reagentes cromogênicos ${ }^{18} \mathrm{e}$ de outras espécies como tetracloreto de titânio ${ }^{19}$, extração por solvente ${ }^{20}$, para gerar cloretos voláteis em atomização em forno de grafite ${ }^{21}$ e para algumas determinações espectrofotométricas nas regiões do ultravioleta, visível e infravermelho.

Os produtos iniciais da sonólise do $\mathrm{CCl}_{4}$ abrem uma nova perspectiva de aplicação analítica para compostos orgânicos halogenados voláteis, devido ao elevado poder oxidante dos radicais formados. Neste trabalho foi avaliada a produção de oxidantes provenientes da degradação sonoquímica do $\mathrm{CCl}_{4}$, empregando sonicador de baixa potência, pela variação do $\mathrm{pH}$ do meio e oxidação dos íons $\mathrm{Fe}^{2+}$ e I- 


\section{PARTE EXPERIMENTAL}

\section{Equipamentos}

As medidas de absorbância foram feitas com cubetas de vidro ou poliestireno com $10 \mathrm{~mm}$ de caminho ótico, fazendo uso de espectrofotômetro Femto 432 (Brasil).

As sonicações das soluções em estudo foram realizadas empregando béquer de borossilicato de $10 \mathrm{~mL}$ (d.i $22 \mathrm{~mm}, 1,25 \mathrm{~mm}$ de espessura da parede e $36 \mathrm{~mm}$ de profundidade). $\mathrm{O}$ equipamento empregado foi um banho ultra-sônico com capacidade para $2 \mathrm{~L}$ da VWR Scientific Instruments, modelo 75D Aquasonic (EUA); freqüência de $40 \mathrm{kHz}$ e potência de $200 \mathrm{~W}$, equipado com temporizador, sistema para controle da temperatura e de descarte do líquido do banho. Medidas de $\mathrm{pH}$ foram feitas com $\mathrm{pHmetro}$ Hanna, Checker 1 (Portugal), equipado com eletrodo de vidro.

Para o preparo da solução saturada de $\mathrm{CCl}_{4}$ foi empregado agitador magnético Fisatom 752A (Brasil) e baguetas revestidas com Teflon. Para purificação da água foi empregado sistema EasyPure RF D7031 (Barnstead, EUA).

\section{Reagentes e soluções}

Todos os reagentes utilizados foram de grau analítico e as soluções foram sempre preparadas com água desionizada. As soluções saturadas de $\mathrm{CCl}_{4}$ foram preparadas imediatamente antes dos experimentos pela adição de gotas de $\mathrm{CCl}_{4}$ (Vetec) em balão volumétrico de $500 \mathrm{~mL}$, completando com água, agitando-se por $1 \mathrm{~min}$. Logo em seguida, foi submetida à agitação magnética por 2 horas, a temperatura ambiente $\left(24 \pm 1^{\circ} \mathrm{C}\right)$. Depois de alguns minutos em repouso, a fase aquosa era separada.

Soluções de iodeto foram preparadas pela diluição de solução estoque $0,1 \mathrm{~mol} \mathrm{~L}^{-1}$ de KI (Quimex), para a avaliação da oxidação sonoquímica deste íon e solução de $\mathrm{IO}_{3}^{-} 0,01 \mathrm{~mol} \mathrm{~L}^{-1}$ foi preparada para oxidação do iodeto a iodo, visando comparar com a produção sonoquímica. Solução $0,25 \mathrm{~g} \mathrm{~L}^{-1}$ de leuco violeta cristal $[4,4$ ',4" metilidinotris $(N, N$-dimetilanilina)], Eastman, foi preparada em meio fosfórico, para avaliar a formação de ácido hipoiodoso.

Soluções de $\mathrm{Fe}^{2+}$ foram obtidas com as diluições adequadas de solução estoque (1000 mg L-1), preparada pela dissolução de $\left(\mathrm{NH}_{4}\right)_{2} \mathrm{Fe}\left(\mathrm{SO}_{4}\right)_{2} \cdot 6 \mathrm{H}_{2} \mathrm{O}$ (Reagen). Soluções aquosas de $\mathrm{Fe}^{3+}$ foram preparadas a partir de $\mathrm{Fe}\left(\mathrm{NO}_{3}\right)_{3} \cdot 9 \mathrm{H}_{2} \mathrm{O}$ (Merck) e para garantir a ausência de $\mathrm{Fe}^{2+}$, foi adicionada solução de $\mathrm{K}_{2} \mathrm{~S}_{2} \mathrm{O}_{8}$, com concentração de $1 \mathrm{~g} \mathrm{~L}^{-1}$. Para os testes do $\mathrm{Fe}^{2+}$ residual e $\mathrm{Fe}^{3+}$ formado foram preparadas soluções de 1,10-fenantrolina e de ácido 5-sulfossalicílico, respectivamente. Para a determinação do cloreto gerado pela sonicação foi preparada solução $0,01 \mathrm{~mol} \mathrm{~L}^{-1} \mathrm{Ag}^{+}$.

\section{Procedimentos}

Para todos os experimentos, o béquer, recoberto com filme de PVC, foi posicionado na zona central do banho ultra-sônico, onde os efeitos de cavitação foram mais pronunciados ${ }^{22}$. A água do banho foi continuamente substituída, empregando sistema de refluxo a vazão de $500 \mathrm{~mL} \mathrm{~min}^{-1}$, garantindo a temperatura da água do banho em $25 \pm 1{ }^{\circ} \mathrm{C}$.

A variação do $\mathrm{pH}$ da água saturada de $\mathrm{CCl}_{4}$ durante a sonicação foi feita para $5 \mathrm{~mL}$ da solução, sendo este parâmetro medido para até 15 min de irradiação. A avaliação da geração de oxidante pela sonicação da solução de $\mathrm{CCl}_{4}$ foi conduzida de duas formas distintas: pela avaliação da oxidação de iodeto e de $\mathrm{Fe}^{2+}$. Assim, 5,00 mL desta solução foram misturados a $250 \mu \mathrm{L}$ de solução $0,01 \mathrm{~mol} \mathrm{~L}^{-1} \mathrm{de}$ $\mathrm{I}^{-}$, sendo sonicados por até $5 \mathrm{~min}$, para posterior extração com
2,00 mL de $\mathrm{CCl}_{4}$, seguindo-se a separação da fase orgânica e, o $\mathrm{I}_{2}$ formado foi determinado pela medida da absorbância em $520 \mathrm{~nm}$. Alternativamente, sem a adição da alíquota de $\mathrm{CCl}_{4}$, a absorbância foi medida em $352 \mathrm{~nm}$, para monitorar a formação de $\mathrm{I}_{3}^{-}$. Para constatar a conversão de $\mathrm{I}^{-}$para HIO, alíquotas de 2,00 mL das soluções sonicadas por diferentes intervalos de tempo foram misturadas com $500 \mu \mathrm{L}$ de solução tampão acetato-ácido acético (pH 4), e $500 \mu \mathrm{L}$ de leuco violeta cristal $0,25 \mathrm{~g} \mathrm{~L}^{-1}$, levando à formação de violeta cristal, o qual apresenta máximo de absorção em $592 \mathrm{~nm}$. As medidas de absorbância foram feitas 2 min após a mistura dos reagentes.

Para investigar a eficiência de oxidação do $\mathrm{Fe}^{2+}$, alíquotas contendo até $12,5 \mu \mathrm{g}$ de $\mathrm{Fe}^{3+}$ ou $\mathrm{Fe}^{2+}$ foram misturadas a $500 \mu \mathrm{L}$ de tampão acetato - ácido acético (ajustando o pH para 2,9) e $500 \mu \mathrm{L}$ de solução $10 \%(\mathrm{~m} / \mathrm{v})$ de ácido 5-sulfossalicílico. Para os experimentos envolvendo soluções de $\mathrm{Fe}^{3+}$ e $\mathrm{Fe}^{2+}$ foram adicionados $500 \mu \mathrm{L}$ de solução $1 \mathrm{~g} \mathrm{~L}^{-1}$ em $\mathrm{S}_{2} \mathrm{O}_{8}^{2-}$ e $1,00 \mathrm{~mL}$ da solução aquosa de $\mathrm{CCl}_{4}$ previamente sonicada por $1 \mathrm{~min}$, respectivamente. Em todas as situações o volume foi levado a 5,00 mL e os sinais foram medidos no comprimento de onda de máxima absorção do complexo $(490 \mathrm{~nm})$.

Para avaliar a taxa de produção de oxidante pela degradação sonoquímica de $\mathrm{CCl}_{4}, 4,00 \mathrm{~mL}$ de água e de solução saturada de $\mathrm{CCl}_{4}$ foram sonicadas por até $50 \mathrm{~s}$, para posterior mistura com 1,00 $\mathrm{mL}$ de solução $40 \mathrm{mg} \mathrm{L}^{-1} \mathrm{Fe}^{2+}$. Experimentos foram também realizados, sonicando 4,00 mL de água e solução de $\mathrm{CCl}_{4} \operatorname{com} 1,00 \mathrm{~mL}$ da mesma solução de $\mathrm{Fe}^{2+}$. Para todas as situações foram determinados os valores de $\mathrm{pH}$, antes e após a sonicação, e a quantidade de $\mathrm{Fe}^{2+}$ residual, empregando o método da 1,10 -fenantrolina $(\lambda=508 \mathrm{~nm})$.

A determinação do cloreto produzido pela sonólise do $\mathrm{CCl}_{4}$ foi realizada empregando método turbidimétrico. Assim, logo após a sonicação de $5,00 \mathrm{~mL}$ de solução aquosa saturada de $\mathrm{CCl}_{4}$ por $3 \mathrm{~min}$ foram adicionados 5,00 mL de solução $0,01 \mathrm{~mol} \mathrm{~L}^{-1}$ de $\mathrm{AgNO}_{3}$ e a leitura foi feita a $540 \mathrm{~nm}$ contra água, após $1 \mathrm{~min}$, sendo os resultados comparados com soluções de referência.

\section{RESULTADOS E DISCUSSÃO}

A solução aquosa de $\mathrm{CCl}_{4}$ irradiada por 4 min com ondas ultrasônicas de $40 \mathrm{kHz}$ apresentou diminuição do $\mathrm{pH}$ da solução de 4,6 para 2,6 (Figura 1), sendo esta variação de $\mathrm{pH}$ um indício da sonólise do $\mathrm{CCl}_{4}$ (eq. 1). Esta inferência pode ser justificada pela produção de $\mathrm{HCl}$ e, possivelmente, de $\mathrm{HClO}$ no meio irradiado, devido à interação do radical cloro com a água (eqs. 2 e 3 ).

$$
\begin{aligned}
& \mathrm{CCl}_{4} \stackrel{)}{\rightarrow} \mathrm{Cl} \cdot+\cdot \mathrm{CCl}_{3} \\
& \mathrm{Cl} \cdot+\mathrm{H}_{2} \mathrm{O} \rightarrow \mathrm{HCl}+\cdot \mathrm{OH} \\
& 2 \mathrm{Cl} \cdot+\mathrm{H}_{2} \mathrm{O} \rightarrow \mathrm{HCl}+\mathrm{HClO}
\end{aligned}
$$

Outra hipótese para a geração desses ácidos está relacionada com a interação do $\mathrm{O}_{2}$ dissolvido no meio com água e $\cdot \mathrm{CCl}_{3}$, o que levaria à produção de fosgênio, o qual se degrada $\mathrm{a} \mathrm{CO}_{2} \mathrm{e} \mathrm{Cl}_{2}$, quando a temperatura está acima de $100{ }^{\circ} \mathrm{C}{ }^{23}$.

Reações envolvendo os radicais $\mathrm{H} \cdot$ e $\mathrm{HO}$, produzidos pela sonólise da água, também poderiam originar estes ácidos. Contudo, este mecanismo é menos provável, visto que a maior pressão de vapor do $\mathrm{CCl}_{4}$ favoreceria a difusão de moléculas deste composto para as cavidades, associando a isto o fato da energia necessária para a clivagem da ligação $\mathrm{C}-\mathrm{Cl}\left(73 \mathrm{kcal} \mathrm{mol}^{-1}\right)$ ser menor que aquela para o rompimento da ligação O-H das moléculas de água $\left(119 \mathrm{kcal} \mathrm{mol}^{-1}\right)$.

A sonicação de solução aquosa saturada de $\mathrm{CCl}_{4}$, para intervalos de tempo da ordem de dezenas de segundos, produz soluções contendo espécies oxidantes, possivelmente $\mathrm{HClO}$ e/ou $\mathrm{Cl}_{2}$, sendo que 


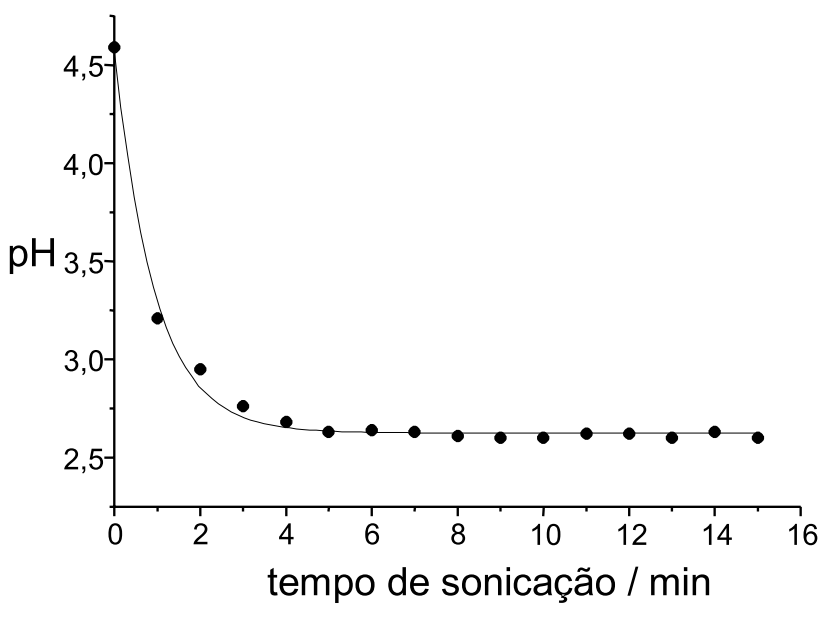

Figura 1. Variação do pH da solução aquosa saturada de $\mathrm{CCl}_{4}$ com o período de sonicação

esta última pode ser produzida pela combinação de radicais cloro, como indicado na equação 4.

$2 \mathrm{Cl} \cdot \rightarrow \mathrm{Cl}_{2}$

Oxidação de iodeto pela sonicação de soluções aquosas de $\mathrm{CCl}_{4}$

A oxidação de $\mathrm{I}^{-}$a $\mathrm{I}_{2}\left(\mathrm{E}^{0}=+0,615 \mathrm{~V}\right)$, provocada pela sonicação, foi um dos primeiros estudos que evidenciaram os efeitos sonoquímicos em soluções. A oxidação ocorre pela ação da hidroxila proveniente da sonólise da água, sendo muito baixo o rendimento da oxidação do iodeto. Contudo, a taxa de conversão de I- para $\mathrm{I}_{2}$ é significativamente aumentada quando a solução de I é preparada em água saturada com $\mathrm{CCl}_{4}{ }^{24}$.

Quando a mistura bifásica água/ $\mathrm{CCl}_{4}$ foi exposta ao ultra-som, as fases aquosa e orgânica imediatamente ganharam uma turvação esbranquiçada, provocada pela formação de emulsão nas duas fases e pela formação de $\mathrm{Cl}_{2}$. Em presença de KI, pôde ser verificada turvação rosada nas duas fases, devido à oxidação do iodeto a iodo molecular e extração do $\mathrm{I}_{2}$ para o meio orgânico.

Quando I' foi sonicado em solução aquosa saturada de $\mathrm{CCl}_{4}$, não foram observadas a coloração rosada e a turvação do meio. Contudo, a oxidação de I- pôde ser caracterizada pela adição posterior de alíquota de $\mathrm{CCl}_{4}$ para extração do $\mathrm{I}_{2}$ e leitura da absorbância em 520 $\mathrm{nm}$. Alternativamente, a oxidação de $\mathrm{I}^{-}$foi realizada monitorando a formação do $\mathrm{I}_{3}^{-}$a $352 \mathrm{~nm}$, ou pela oxidação do leuco violeta cristal.

$\mathrm{O}$ primeiro procedimento para caracterização de $\mathrm{I}_{2}$ formado foi baseado na extração deste com $\mathrm{CCl}_{4}$, o qual origina uma solução com máximo de absorção a $520 \mathrm{~nm}$. A curva representada na Figura 2 mostra que a oxidação do iodeto foi dependente do período de irradiação. Para 2 min de sonicação, quantidades suficientes de $\mathrm{Cl}_{2} \mathrm{e}$ de radicais cloro foram geradas para produzir $\mathrm{I}_{2}$ a partir de solução de iodeto $0,476 \mathrm{mmol} \mathrm{L}^{-1}$. Assim, para este período de sonicação, obteve-se conversão de $97,6 \%$ do I- para $\mathrm{I}_{2}$. Todavia, pôde ser também verificada a tendência de diminuição do $\mathrm{I}_{2}$ presente no meio para períodos de sonicação maiores que 2 min (Figura 2), possivelmente devido à volatilização ou oxidação desta espécie.

No segundo procedimento, para a avaliação da formação de $\mathrm{I}_{3}^{-}$ que absorve em $352 \mathrm{~nm}$, constatou-se que esta espécie prevaleceu para tempos de sonicação inferiores a 1 min (Figura 3). Para tempos de irradiação maiores que $1 \mathrm{~min}$, o I' presente continua sendo convertido a $\mathrm{I}_{2}$, e a solução adquire coloração castanha, característica do $\mathrm{I}_{2}$, que não absorve em $352 \mathrm{~nm}$.

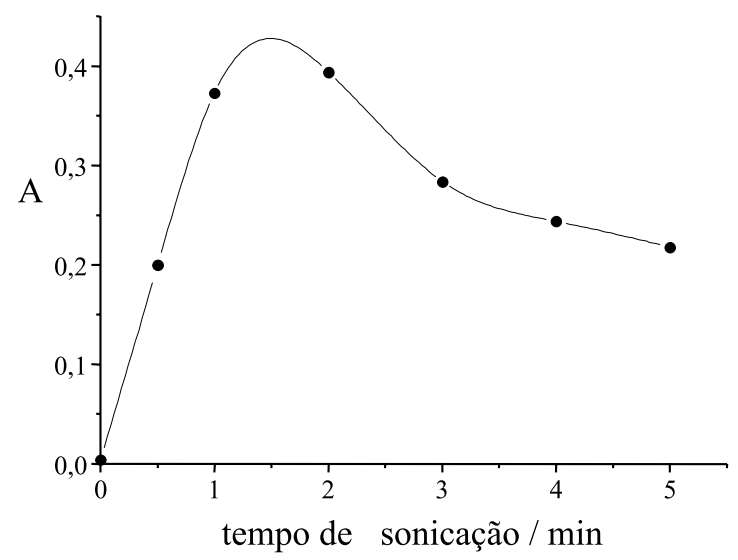

Figura 2. Produção de $I_{2}$ pela oxidação de iodeto em solução aquosa saturada de $\mathrm{CCl}_{4}$ com o período de sonicação

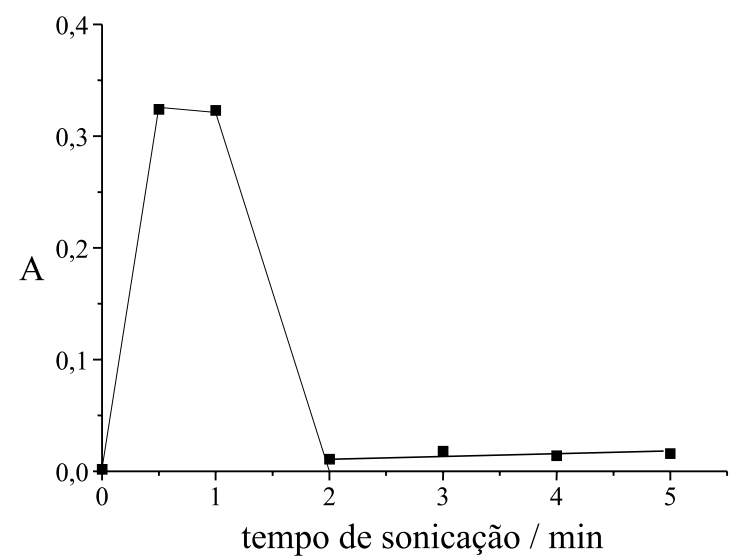

Figura 3. Formação de $I_{3}^{-}$com o intervalo de tempo de sonicação em solução de iodeto saturada com $\mathrm{CCl}_{4}$

O terceiro procedimento foi baseado na oxidação seletiva de leuco violeta cristal pelo HIO para violeta cristal, que apresenta máxima absorção a $592 \mathrm{~nm}\left(\varepsilon \cong 1 \times 10^{5} \mathrm{~L} \mathrm{~mol}^{-1} \mathrm{~cm}^{-1}\right)^{25,26}$, sendo comprovada a transição do iodo do estado de oxidação -1 para +1 , desde os primeiros instantes de sonicação (Figura 4). Esta série de experimentos

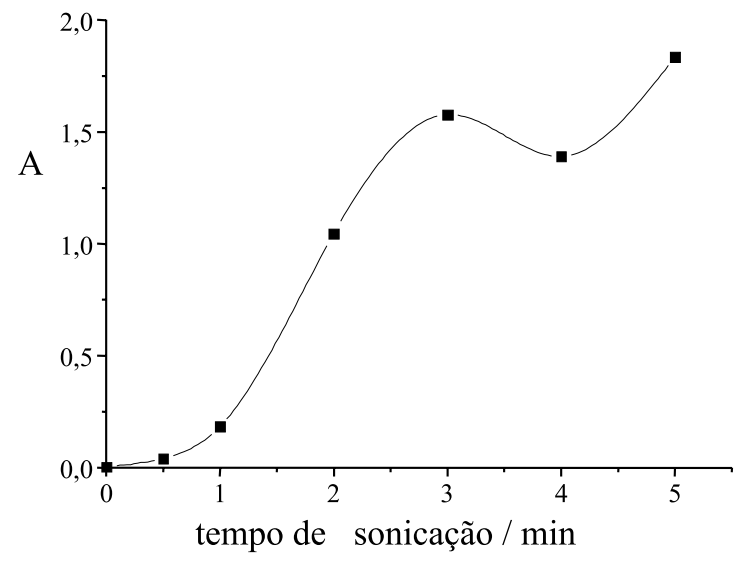

Figura 4. Produção de HIO com o período de sonicação em solução de iodeto saturada com $\mathrm{CCl}_{4}$ 
comprovou que a oxidação de iodeto em solução aquosa de $\mathrm{CCl}_{4}$ sob ação de ondas ultra-sônicas ocorreu por conversões sucessivas para estados de oxidação mais elevados, sendo que para quaisquer períodos de sonicação estudados co-existiram espécies de iodo em diferentes estados de oxidação.

\section{Oxidação sonoquímica de $\mathrm{Fe}^{2+}$}

A exposição de uma solução aquosa de $\mathrm{Fe}^{2+}$ às ondas ultra-sônicas resulta em oxidação deste íon $\left(\mathrm{E}_{\mathrm{Fe}}^{0}{ }^{3+} / \mathrm{Fe}{ }^{2+}=+0,771 \mathrm{~V}\right)$. Nestas condições, $\mathrm{o} \mathrm{Fe}^{2+}$ interage com o radical $\cdot \mathrm{OH}$ gerado pela sonólise da água para formar um íon $\mathrm{Fe}^{3+}$ e o íon hidróxido ${ }^{27,28}$, segundo a equação 5:

$\mathrm{Fe}^{2+}+\cdot \mathrm{OH} \rightarrow \mathrm{Fe}^{3+}+\mathrm{OH}^{-}$

A sonicação prévia de água, para posterior mistura com a solução de $\mathrm{Fe}^{2+}$, não garantiu a oxidação expressiva destes íons no intervalo de tempo estudado. Por sua vez, a solução oxidante preparada pela sonicação da solução aquosa saturada de $\mathrm{CCl}_{4}$ mostrou-se eficiente para oxidação quantitativa de $\mathrm{Fe}^{2+}$. $\mathrm{O} \mathrm{Fe}^{3+}$ produzido após mistura com a solução aquosa de $\mathrm{CCl}_{4}$ sonicada por $1 \mathrm{~min}$ foi determinado em $490 \mathrm{~nm}$, fazendo uso da reação de formação de complexo com ácido 5-sulfossalicílico. Os valores de absorbância para diferentes concentrações de $\mathrm{Fe}^{3+}$ produzidos pela ação sonoquímica são apresentados na Tabela 1, na qual podem ser comparados com aqueles obtidos com soluções de $\mathrm{Fe}^{3+}$ nas mesmas concentrações.

Tabela 1. Comparação dos valores de absorbância de soluções aquosas de $\mathrm{Fe}^{3+}$ e de soluções aquosas de $\mathrm{Fe}^{2+}$ misturadas com o produto da sonicação de solução aquosa saturada com $\mathrm{CCl}_{4}$ por 2 min, após mistura com solução de ácido 5-sulfossalicílico e leitura em $490 \mathrm{~nm}$

\begin{tabular}{ccc}
\hline Quantidade de & \multicolumn{2}{c}{ Absorbância } \\
\cline { 2 - 3 } $\mathrm{Fe}(\mu \mathrm{g})$ & $\mathrm{Fe}^{3+}$ & $\mathrm{Fe}^{2+} / \mathrm{US}^{*}$ \\
\hline 0 & 0,003 & 0,003 \\
2,5 & 0,021 & 0,021 \\
5,0 & 0,040 & 0,038 \\
7,5 & 0,054 & 0,056 \\
10,0 & 0,071 & 0,071 \\
12,5 & 0,085 & 0,086
\end{tabular}

* $\mathrm{Fe}^{2+} / \mathrm{US}=$ Soluções de $\mathrm{Fe}^{3+}$ produzidas pela adição de solução aquosa saturada com $\mathrm{CCl}_{4}$ sonicada por 1 min a soluções de $\mathrm{Fe}^{2+}$.

Os perfis de oxidação de $\mathrm{Fe}^{2+}$ para diferentes tempos de sonicação e em diferentes condições experimentais são apresentados na Figura 5. Com a sonicação prévia da água por até $50 \mathrm{~s}$ (curva a, Figura 5) não houve oxidação significativa do $\mathrm{Fe}^{2+}(<1,3 \%)$, indicando baixa produção de oxidante nestas condições experimentais. Contudo, quando o íon metálico está presente durante a insonação, a oxidação para $\mathrm{Fe}^{3+}$ é mais pronunciada (curva b, Figura 5), confirmando a hipótese da participação do radical hidroxila, proveniente da sonólise da água, no mecanismo de oxidação do $\mathrm{Fe}^{2+}$.

Para o caso da adição de solução de $\mathrm{Fe}^{2+}$ à água saturada com $\mathrm{CCl}_{4}$, previamente sonicada, houve um significativo aumento da concentração de $\mathrm{Fe}^{3+}$ (curva c, Figura 5), sendo que após 40 s de sonicação da solução de $\mathrm{CCl}_{4}, 40 \mu \mathrm{g}$ de $\mathrm{Fe}^{2+}$ foram quantitativamente convertidos a $\mathrm{Fe}^{3+}$. A conversão para $\mathrm{Fe}^{3+}$ pela sonicação do $\mathrm{Fe}^{2+}$ presente na solução aquosa de $\mathrm{CCl}_{4}$ mostrou-se ainda mais eficaz (curva d, Figura 5), possivelmente devido à interação direta dos radicais cloro com o íon metálico (eq. 6).

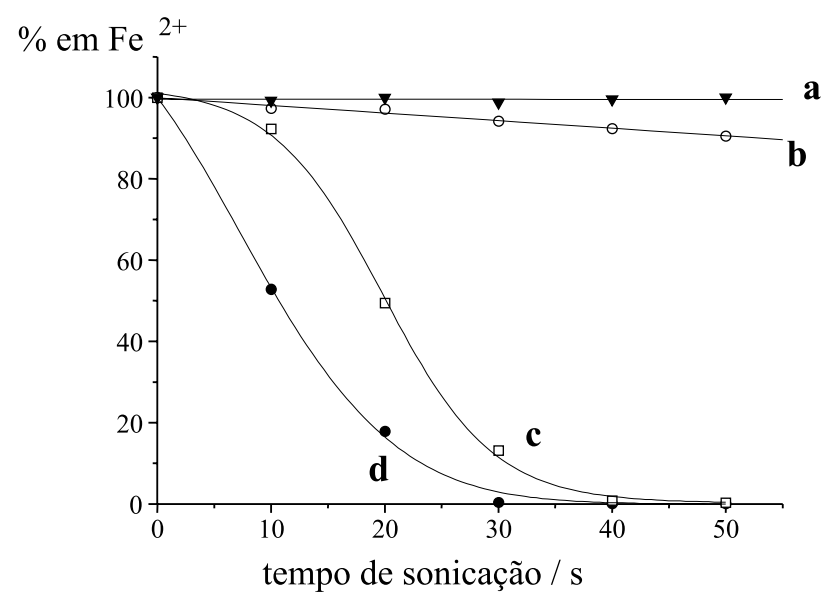

Figura 5. $\mathrm{Fe}^{2+}$ residual para diferentes tempos e condições de sonicação. (a) sonicação prévia de água e posterior mistura com solução de $\mathrm{Fe}^{2+}$; (b) sonicação da solução aquosa de $\mathrm{Fe}^{2+}$; (c) sonicação prévia de água saturada com $\mathrm{CCl}_{4}$ para posterior adição da solução de $\mathrm{Fe}^{2+}$; (d) sonicação de solução aquosa de $\mathrm{Fe}^{2+}$ saturada com $\mathrm{CCl}_{4}$

$\mathrm{Fe}^{2+}+\cdot \mathrm{Cl} \rightarrow \mathrm{Fe}^{3+}+\mathrm{Cl}^{-}$

\section{Taxa de formação de oxidantes}

Como o iodo pode estar presente em solução em diversos estados de oxidação, estimativas das taxas de produção de oxidantes, oriundos da sonólise do $\mathrm{CCl}_{4}$, foram realizadas baseadas nos estudos realizados com soluções de $\mathrm{Fe}^{2+}$. Assim, pela monitoração da concentração do $\mathrm{Fe}^{2+}$ residual e do $\mathrm{pH}$, nas condições de sonicação anteriormente citadas, foi possível determinar as taxas de formação e degradação de algumas espécies, bem como propor equações para estabelecer as quantidades de $\mathrm{Fe}^{2+}$ oxidado $\mathrm{e}_{3} \mathrm{O}^{+}$produzido para diferentes tempos de sonicação (Tabela 2). Pela Tabela 2 pode ser constatado que não há diferenças significativas da formação de $\mathrm{H}^{+} \mathrm{e}$ oxidação de $\mathrm{Fe}^{2+}$ para a sonicação conjunta, ou não, da solução de $\mathrm{Fe}^{2+}$ com água saturada com $\mathrm{CCl}_{4}$. Por outro lado, o rendimento associado à produção de $\mathrm{H}^{+} \mathrm{e}$ oxidação de $\mathrm{Fe}^{2+} \mathrm{a} \mathrm{Fe}$ é maior quando solução saturada com $\mathrm{CCl}_{4}$ está envolvida no sistema sob sonicação.

A oxidação de $\mathrm{Fe}^{2+} \mathrm{e}$ a diminuição do $\mathrm{pH}$ nesses experimentos são creditadas à ação do radical cloro sobre $\mathrm{o} \mathrm{Fe}^{2+} \mathrm{e} \mathrm{H}_{2} \mathrm{O}$, respectivamente. Em ambas reações, a relação estequiométrica é 1:1 (eqs. $6 \mathrm{e}$ 2), logo a taxa de formação do radical cloro deve ser a soma da taxa de oxidação de $\mathrm{Fe}^{2+}$ à de formação de $\mathrm{H}_{3} \mathrm{O}^{+}$, ou seja, $0,107 \mu \mathrm{mol} \mathrm{s} \mathrm{s}^{-1}$.

A oxidação de $\mathrm{Fe}^{2+}$ para sistema envolvendo a sonicação prévia da solução de $\mathrm{CCl}_{4}$ seguida da adição de solução deste íon, resulta da

Tabela 2. Equações semi-empíricas para cálculos da quantidade de matéria (Q, mmol) consumida de $\mathrm{Fe}^{2+}$ e produzida de $\mathrm{H}^{+}$com o tempo de sonicação para os diferentes sistemas irradiados.

\begin{tabular}{lcc}
\hline Sistemas sonicados & $\begin{array}{c}\mathrm{H}^{+} \text {produzido } \\
(\mu \mathrm{mol})\end{array}$ & $\begin{array}{c}\mathrm{Fe}^{2+} \text { consumido } \\
(\mu \mathrm{mol})\end{array}$ \\
\hline $\begin{array}{l}\text { Solução aquosa de } \mathrm{Fe}^{2+} \\
\text { saturada com } \mathrm{CCl}_{4}\end{array}$ & $-0,012+0,083 . \mathrm{t}_{\mathrm{s}}$ & $0,70-0,024 . \mathrm{t}_{\mathrm{s}}$ \\
$\begin{array}{l}\text { Solução aquosa de } \mathrm{CCl}_{4} \\
\text { e adição de solução de } \mathrm{Fe}^{2+}\end{array}$ & $-0,043+0,064 . \mathrm{t}_{\mathrm{s}}$ & $0,78-0,028 . \mathrm{t}_{\mathrm{s}}$ \\
${\text { Solução aquosa de } \mathrm{Fe}^{2+}}$ & $-0,026+0,027 . \mathrm{t}_{\mathrm{s}}$ & $0,71-0,0013 . \mathrm{t}_{\mathrm{s}}$ \\
\hline
\end{tabular}


interação entre $\mathrm{o} \mathrm{Cl}_{2}$, gerado durante a irradiação, com o íon ferroso. Desta forma, sabendo-se a relação estequiométrica envolvida na reação de $\mathrm{Fe}^{2+}$ com $\mathrm{Cl}_{2}(2: 1)$, foi possível estabelecer a taxa de geração de $\mathrm{Cl}_{2}$ e do radical cloro. Desta forma, a taxa de formação do $\mathrm{Cl}_{2}$ será a metade da taxa de oxidação do $\mathrm{Fe}^{2+}$, ou seja, $0,014 \mu \mathrm{mol} \mathrm{s}{ }^{-1}$. Como são necessários dois radicais cloro para originar uma molécula de $\mathrm{Cl}_{2}$, a taxa de formação de $\cdot \mathrm{Cl}$ será a soma do dobro da taxa de formação de $\mathrm{Cl}_{2}$ com a de formação de $\mathrm{H}^{+}$, ou seja, 0,092 $\mu \mathrm{mol} \mathrm{s}{ }^{-1}$.

A função linear que relaciona a geração de oxidante com o tempo de sonicação, nas condições estabelecidas, foi válida para intervalos de tempo de irradiação até $50 \mathrm{~s}$, uma vez que com o aumento do período de sonicação diminui a quantidade do $\mathrm{CCl}_{4}$. Desta forma, para intervalos de tempo de sonicação maiores, da ordem de centenas de segundos, seria esperada uma brusca queda na taxa de geração de radicais. Deve ser mencionado que respostas diferentes podem ser obtidas empregando diferentes equipamentos, visto que a zona de maior incidência de ultra-sons, bem como a geometria, potência e frequência dos sonicadores e a amplitude das ondas ultrasônicas devem variar, mesmo para equipamentos de mesma marca e modelo.

\section{Produção sonoquímica de cloreto}

A avaliação da produção de íons $\mathrm{Cl}^{-}$em soluções aquosas de $\mathrm{CCl}_{4}$ irradiadas está intimamente relacionada ao aumento da acidez do meio, dada a provável formação de $\mathrm{HCl}$. A quantidade de cloreto produzido após 3 min de sonicação da solução saturada de $\mathrm{CCl}_{4}$ foi igual a $12,19 \mu \mathrm{mol}$.

Considerando-se que a produção de $\mathrm{H}_{3} \mathrm{O}^{+}$é igual à de $\mathrm{Cl}^{-}$e, fazendo uso da taxa de formação de $\mathrm{H}_{3} \mathrm{O}^{+}$, referente à sonicação prévia da solução de $\mathrm{CCl}_{4}$ (Tabela 2), a quantidade calculada de cloreto que deveria ser produzida após 3 min de sonicação é 11,52 $\mu$ mol. Desta forma foi confirmado que a variação de $\mathrm{pH}$ pode ser utilizada para monitorar a sonólise do $\mathrm{CCl}_{4}$ em solução aquosa, ou seja, sua degradação sonoquímica.

\section{CONCLUSÕES}

A exposição de solução aquosa saturada com $\mathrm{CCl}_{4}$ a ondas ultrasônicas leva à degradação do composto orgânico pela clivagem das ligações $\mathrm{C}-\mathrm{Cl}$, gerando radicais $\cdot \mathrm{Cl}$, que podem interagir com moléculas de água, produzindo $\mathrm{HCl}$; oxidar outras espécies presentes no meio durante a irradiação; bem como, os radicais podem ser combinados, formando $\mathrm{Cl}_{2}$. Nos estudos realizados pôde ser constatado que reações de oxidação conduzidas pela sonicação de solução aquosa de $\mathrm{CCl}_{4}$ apresentaram maior rendimento que aquelas com água. Possivelmente pela maior quantidade de radicais livres produzidos durante a sonólise do $\mathrm{CCl}_{4}$.

Além do processo poder ser empregado para a determinação de espécies químicas, a produção in situ de oxidantes pela sonicação de soluções pode ser uma alternativa para o preparo de amostras, reduzindo a quantidade de reagentes e o descarte para o ambiente.
Apesar da taxa de produção de oxidante pela sonólise do $\mathrm{CCl}_{4}$ não ser elevada, devido à baixa solubilidade deste solvente orgânico em água, é possível a aplicação em procedimentos de escala reduzida. Processos para a re-saturação da solução aquosa com $\mathrm{CCl}_{4}$, visando a produção de maior quantidade de cloro, estão sendo estudados.

\section{AGRADECIMENTOS}

S. S. Borges e M. Korn agradecem o apoio financeiro do Conselho Nacional de Desenvolvimento Científico e Tecnológico (CNPq) e da Superintendência de Apoio ao Desenvolvimento Científico e Tecnológico da Secretaria de Planejamento, Ciência e Tecnologia do Estado da Bahia (CADCT/SEPLANTEC). Os autores são gratos à Eastman do Brasil pela doação de reagentes.

\section{REFERÊNCIAS}

1. Mason, T. J. ; Lorimer, J. P.; Sonochemistry: Theory, Applications and Uses of Ultrasound in Chemistry, Ellis Horwood: Chichester, 1988, p. 4.

2. Suslick, K. S.; Science 1990, 247, 1439.

3. Suslick, K. S.; Sci. Am. 1989, 2, 80.

4. Suslick, K.S.; Hammerton, D. A.; Cline Jr., R. E.; J. Am. Chem. Soc. 1986, 108, 5641.

5. Hua, I.; Hoffmann, M. R.; Environ. Sci. Technol. 1997, 31, 2237.

6. Fitzgerald, M. E.; Griffing, V.; Sullivan J.; J. Chem. Phys. 1956, 25, 926.

7. Wakeford, C. A.; Blackburn, R.; Lickiss, P. D.; Ultrason. Sonochem. 1999, 6,141 .

8. Suslick, K. S.; Choe, S. B.; Cichowlas, A. A.; Grinstaff, M. W.; Nature 1991, 353, 414.

9. Doktycz, S. J.; Suslick, K. S.; Science 1990, 247, 1067.

10. Weissler, A.; J. Am. Chem. Soc. 1950, 171, 1007.

11. Hua, I.; Hoffmann, M. R.; Environ. Sci. Technol. 1996, 30, 864.

12. Francony, A.; Pétrier, C.; Ultrason. Sonochem. 1996, 3, 77.

13. Alippi, A.; Cataldo, F.; Galbato, A.; Ultrasonics 1992, 30, 148.

14. Bhatnagar, A.; Cheung, H. M.; Environ. Sci. Technol. 1994, 28, 1481.

15. Cheung, H. M.; Bhatnagar, A.; Jansen, G.; Environ. Sci. Technol. 1991, 25,510 .

16. Orzechowska, G. E.; Poziomek, E. I.; Hodge, V. F.; Engelmann, W. H.; Environ. Sci. Technol. 1995, 29, 1373.

17. Hung, H. M.; Hoffmann, M. R.; Environ. Sci. Technol. 1998, 32, 3011.

18. Cheng, K. L.; Ueno, K.; Imamura, T.; Handbook of Organic Analytical Reagents, CRC Press: Florida, 1982, p. 331.

19. Carvalho, R. M.; Kubota, L. T.; Rohwedder, J. J. R.; Csöregi, E.; Gorton, L.; J. Electroanal. Chem. 1998, 457, 83.

20. Ninan, S.; Varadarajan, A.; Jadhav, S. B.; Kulkarni, A. J.; Malve, S. P.; Spectrochim. Acta A 1999, 55, 825.

21. Kántor, T.; Spectrochim. Acta B 2000, 55, 431.

22. Nascentes, C. C.; Korn, M.; Sousa, C. S.; Arruda, M. A. Z.; J. Braz. Chem. Soc. 2001, 12, 57.

23. Battin-Leclerc, F.; Baronnet, F.; Paternotte, G.; Leclerc, J. P.; Gourhan, R.; J. Anal. Appl. Pyrolysis 2000, 53, 95.

24. Weissler, A; Cooper, H. W.; Snyder, S.; J. Am. Chem. Soc. 1950, 72, 1769.

25. Lambert, J. L.; Hatch, G. L.; Moiser, B.; Anal. Chem. 1975, 47, 915.

26. Hatch, G. L.; Anal. Chem. 1984, 56, 2238.

27. Sehgal, C.; Sutherland, R. G.; Verral, R. E.; J. Phys. Chem. 1980, 84, 2920.

28. Él'Piner, I. E.; Ultrasound: Physical, Chemical, and Biological Effects, Consultants Bureau: New York, 1964, p. 25. 